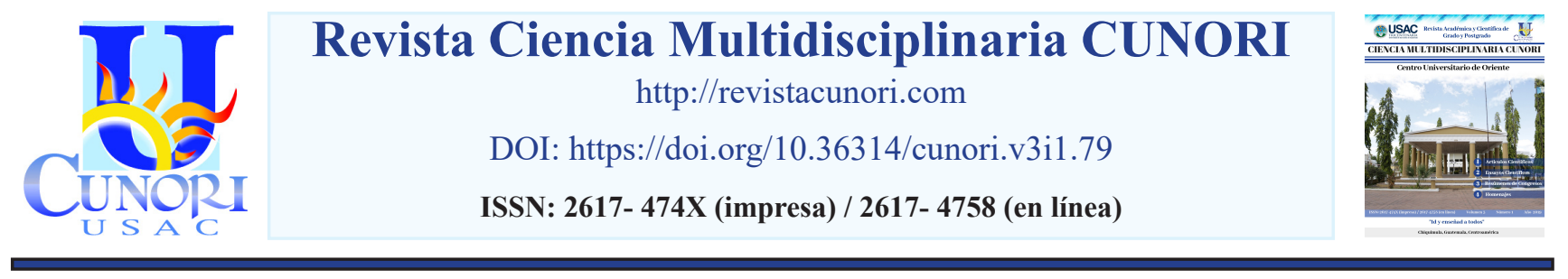

Como citar el artículo

Figueroa, W., Mazariegos, E., Ramos, S., (2019). Caracterización de pacientes con fracturas producidas por proyectil de arma de fuego. Revista Ciencia Multidisciplinaria CUNORI, 3(1), 51-56. DOI: https://doi.org/10.36314/cunori.v3i1.79

\title{
Caracterización de pacientes con fracturas producidas por proyectil de arma de fuego
}

\section{Characterization of patients with fractures produced by firearm projectile}

\author{
Welner Figueroa*, Edvin Mazariegos, Silver Ramos \\ Centro Universitario de Oriente (CUNORI), Universidad de San Carlos de Guatemala
}

Recibido: 08 de agosto de 2018 / Revisión: 12 de septiembre de 2018 / Aceptado: 21 de enero de 2019

Disponible en internet el 30 de agosto de 2019

\author{
*Autor para correspondencia. \\ Resumen \\ Correo electrónico: welner_figue27@hotmail.com
}

\begin{abstract}
T as fracturas producidas por proyectil de arma de fuego son definidas como lesiones óseas de alta energía con destrucción de —ejidos blandos y alto grado de contaminación, según clasificación de fracturas expuestas; pueden llegar a provocar complicaciones como discapacidades temporales o permanentes. Se realizó un estudio descriptivo retrospectivo que caracterizó clínica y epidemiológicamente a 82 pacientes con fracturas producidas por proyectil de arma de fuego en el Hospital Regional de Zacapa, durante enero de 2013 a diciembre de 2017. Los años con mayor frecuencia de casos fueron en 2016 y 2017 en $26 \%$ y 27\%, respectivamente. El género masculino predominó en $89 \%$, en edades de $19-40$ años en $70 \%$, refirieron ser comerciantes en $26 \%$, procedentes del municipio de Zacapa en $46 \%$, la principal circunstancia fue enfrentamiento de personas con armas de fuego en $32 \%$ y horarios de 18:01-24:00 en 54\% de casos. Predominó el tipo de lesión en miembros inferiores en 56\%, con mayor frecuencia en fracturas de fémur en $20 \%$, el tratamiento quirúrgico se realizó en el $90 \%$ de casos y se presentó complicaciones en $37 \%$, donde por el tipo de fractura y complicaciones fueron incapacitados para trabajar por más de un mes el $54 \%$ y permanentemente el 2\%, según el Congreso de la República de Guatemala. Estos datos podrían atribuir que el sexo masculino en estas edades se expone a trabajos ocasionalmente en horarios nocturnos y podrían necesitar utilizar armas de fuego como defensa o de ataque, además, de ser víctimas de asaltos, violencia o balas perdidas.
\end{abstract}

Palabras clave: caracterización clínica y epidemiológica, fracturas, proyectil de arma de fuego, fémur, paciente

\section{Abstract}

$\mathrm{T}$ he fractures produced by a firearm projectile are defined as high-energy bone injuries with destruction of soft tissues and a high degree of contamination, according to the classification of the exposed fractures; they can lead to complications such as temporary or permanent disabilities. A retrospective descriptive study that characterized 82 patients with fractures produced by firearm projectile in the Regional Hospital of Zacapa between January 2013 and December 2017. The most frequent years of cases were in 2016 and 2017 at $26 \%$ and $27 \%$, respectively. The male gender prevailed in $89 \%$, in ages of $19-40$ years in $70 \%$, they referred to being traders in $26 \%$, coming from the municipality of Zacapa in $46 \%$, the main circumstance was the confrontation of people with weapons of fire in 32\% and schedules from 18:01-24:00 in 54\% of cases. The type of injury in the lower limbs predominated in $56 \%$, with more frequency in femoral fractures in $20 \%$, surgical treatment in $90 \%$ of the cases and complications in $37 \%$, where according to the type of fracture and complications were unable to work for more than a month $54 \%$ and permanently $2 \%$ according to the Congress of the Republic of Guatemala. These data could attribute that male gender in these ages is exposed to work occasionally at night and may need to use firearms as a defense or attack, as well as being victims of assault, violence or stray bullets.

Keywords: clinical and epidemiological characterization, fractures, projectile of firearm, femur, patient 


\section{Introducción}

Las fracturas por proyectil de arma de fuego se pueden definir como fracturas abiertas y complejas en términos de choque óseo con pérdidas de tejidos blandos (Kuyigwa, et al. 2015:122). Teniendo en cuenta que la velocidad del proyectil debe ser mayor a $61 \mathrm{~m} / \mathrm{s}$ para poder producir una fractura ósea (Pérez, 2003:26). Este tipo de lesión traumática, dependiendo de la región anatómica y el segmento óseo afectado, puede llegar a provocar diferentes tipos de complicaciones como discapacidades, disminuyendo la calidad de vida del paciente. Una fractura por proyectil de arma de fuego es una fractura expuesta de alta energía con alto grado de contaminación, varios estudios demuestran que el calor generado durante el disparo no hace estéril a la bala (Rodríguez, 2011:148). Las personas en quienes no se produce una muerte inmediata por estas lesiones llegan a sufrir complicaciones como discapacidades temporales o permanentes, siendo común el uso de estas armas en la región oriente de este país.

No existe una estadística real de este tipo de fracturas pero sí se conocen reportes y publicaciones que mencionan que el número de pacientes va en aumento por factores como jóvenes en pandillas, consumo y tráfico de drogas, desintegración familiar y casos en que menores de edad manipulan de manera inexperta un arma de fuego que es propiedad de un adulto (Palma, 2011:136).

Por lo descrito anteriormente se decide realizar un estudio con el objetivo de caracterizar clínica y epidemiológicamente a los pacientes ingresados con el diagnóstico de fracturas producidas por proyectil de arma de fuego en el Hospital Regional de Zacapa durante el período de enero del año 2013 a diciembre del año 2017; se determinó que este tipo de fracturas predominan en el sexo masculino, edades de 19 a 40 años, comerciantes, principalmente del municipio de Zacapa.

\section{Materiales y métodos}

Se realizó un estudio descriptivo retrospectivo sobre la caracterización clínica y epidemiológica de pacientes con fracturas producidas por proyectil de arma de fuego en el Hospital Regional de Zacapa, durante el período de enero de 2014 a diciembre de 2017. Se elaboró una boleta de recolección de datos, donde se incluyeron 82 expedientes clínicos (Universo) que cumplieran con los criterios de inclusión. Se solicitó la aprobación a las autoridades y al comité de ética del Hospital Regional de Zacapa el debido permiso para poder acceder y revisar los expedientes clínicos dentro del departamento de estadística del hospital descrito, después de recopilar la información de las boletas, se tabularon los datos obtenidos y se procedió a realizar el análisis estadístico por medio de gráficas en el programa de Microsoft Office Excel, para representar los resultados obtenidos a través de la investigación, con frecuencias simples y porcentajes. La información obtenida por medio de las boletas de recolección de datos se manejó confidencialmente, sin mencionar nombres ni apellidos de los pacientes. 


\section{Resultados}

Año de consulta de los pacientes con diagnóstico de fracturas producidas por proyectil de arma de fuego

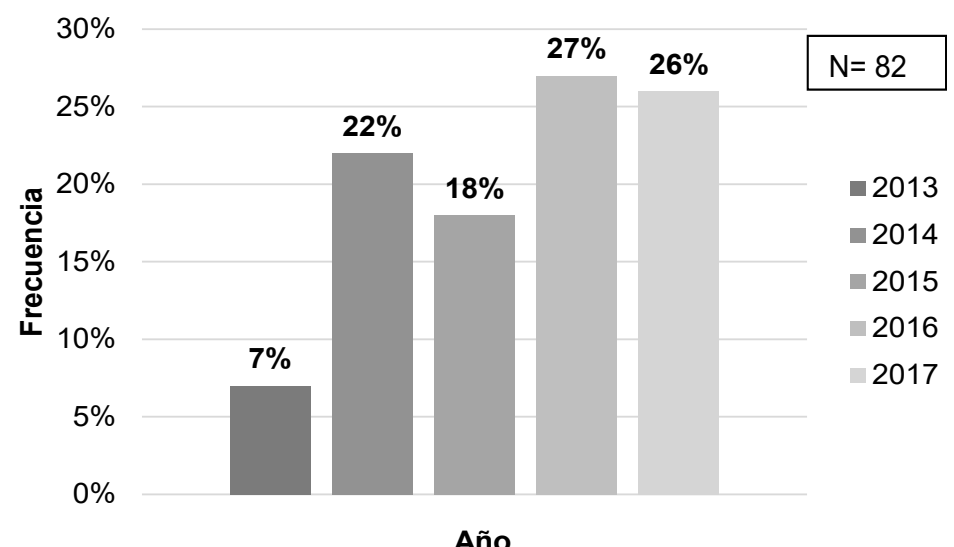

Figura 1. Distribución según el año de consulta de los pacientes con diagnóstico de fracturas producidas por proyectil de arma de fuego, ingresados en el Hospital Regional de Zacapa durante el período de enero de 2013 a diciembre de 2017.

La mayor frecuencia por año se presentó en un 27\% (22) en el año 2016, seguido de un 26\% (21) en el año 2017 y en $22 \%$ (18) en el año 2014.

Género de los pacientes con diagnóstico de fracturas producidas por proyectil de arma de fuego

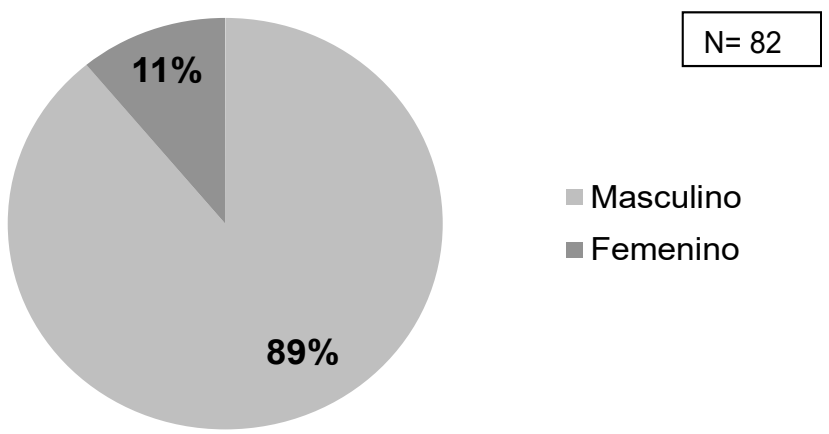

Figura 2. Distribución según el género de los pacientes con diagnóstico de fracturas producidas por proyectil de arma de fuego, ingresados en el Hospital Regional de Zacapa durante el período de enero de 2013 a diciembre de 2017.

El género con más frecuencia se presentó en un 89\% (73) en el género masculino y solo en un 11\% (9) en el género femenino. 
Segmento óseo con lesión, en los pacientes con diagnóstico de fracturas producidas por proyectil de arma de fuego

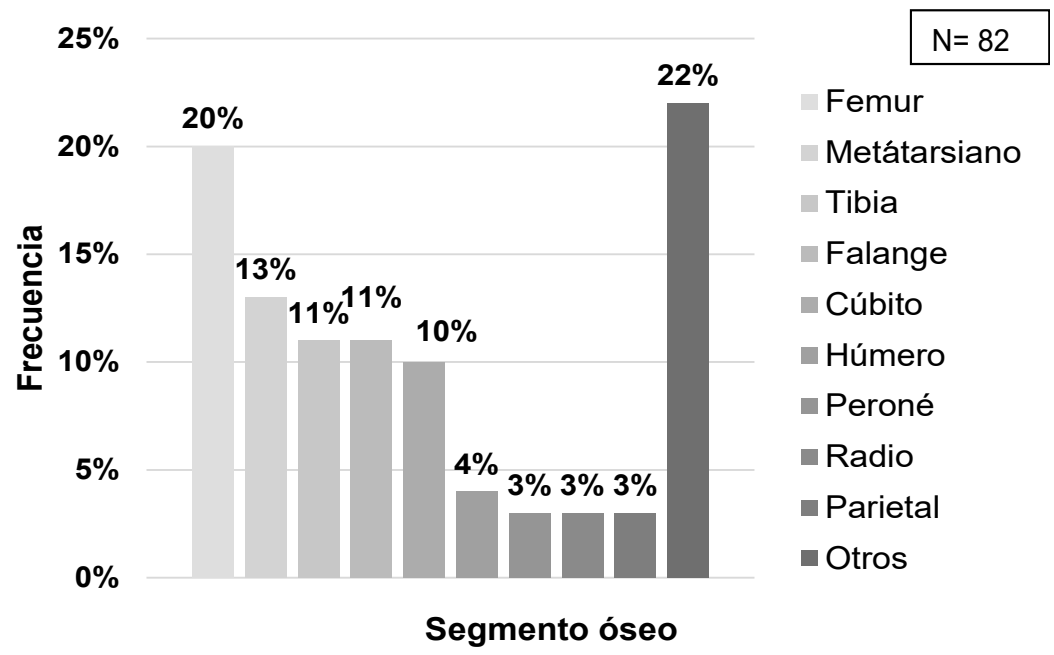

Figura 3. Distribución según el segmento óseo con lesión, en los pacientes con diagnóstico de fracturas producidas por proyectil de arma de fuego, ingresados en el Hospital Regional de Zacapa durante el período de enero de 2013 a diciembre de 2017.

El 20\% (19) correspondió a las fracturas de fémur, seguido por el 13\% (12) correspondiendo a las fracturas de metatarsianos, continuando con un 11\% (10) en fracturas de tibia y de falange, cada una.

Clasificación de lesiones graves por el Congreso de la República de Guatemala, en los pacientes con diagnóstico de fracturas producidas por proyectil de arma de fuego

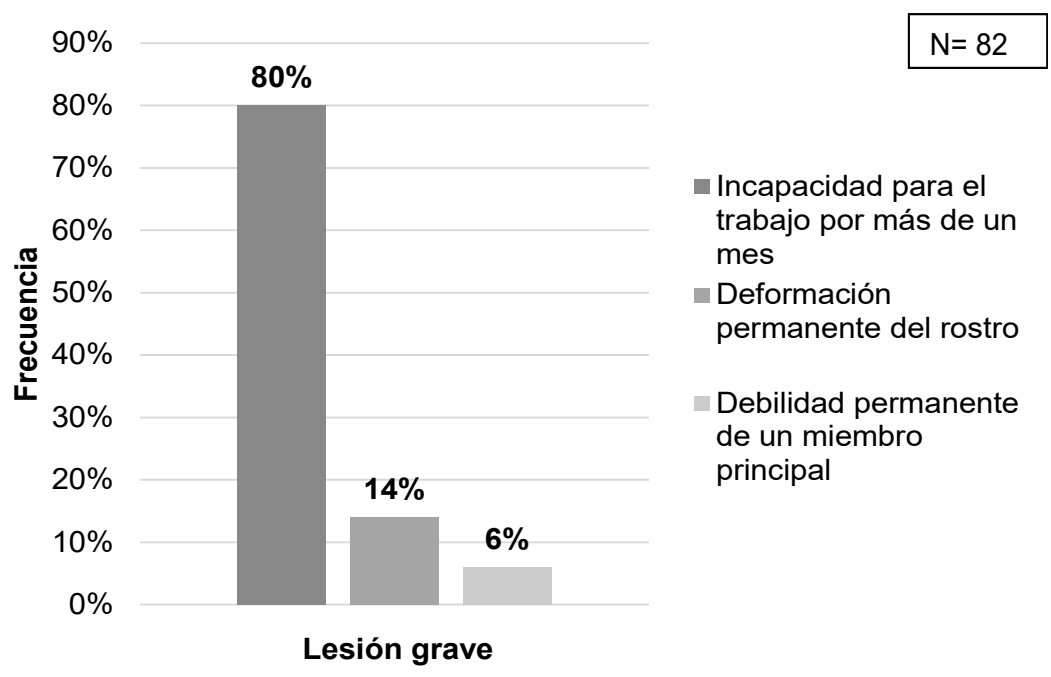

Figura 4. Distribución según clasificación de lesiones graves por el Congreso de la República de Guatemala, en los pacientes con diagnóstico de fracturas producidas por proyectil de arma de fuego, ingresados en el Hospital Regional de Zacapa durante el período de enero de 2013 a diciembre de 2017.

Por el tipo de fracturas y complicaciones el 80\% (44), fue incapacitado para trabajar por más de un mes, el 14\% (8) presentó una deformidad permanente del rostro y el 6\% (3) presentó debilidad permanente de un miembro principal. 


\section{Discusión}

De un total de 82 pacientes identificados en el estudio, se determinó que respecto a los años con mayor frecuencia de este tipo de fracturas predominaron el 2016 con 27\% (22) y el 2017 con 26\% (21), teniendo en cuenta que según la Dirección General de Control de Armas y Municiones en Guatemala, en el año 2,009, se registraron 3,979 armas de fuego por cada 100,000 habitantes, es decir un arma por cada 25 guatemaltecos (Baires, 2014); se evidencia con estos datos que el uso de estas armas con y sin licencia para portarlas ha aumentado en los últimos dos años en esta parte del país; con registros que Centroamérica es una de las tres regiones del mundo en que se producen más muertes por la violencia armada (CICIG, 2009:13).

Predominó el sexo masculino en 89\% (73), en edades de 19-40 años en 70\% (57), estado civil soltero en $42 \%$ (34), refiriendo ser comerciantes en 26\% (21), procedentes del municipio de Zacapa en $46 \%$ (38), con la principal circunstancia de enfrentamiento de personas con armas de fuego en $32 \%$ (26), en horarios de 18:01-24:00 en 54\% (44), concordando estos datos con un estudio realizado en el oriente del país en el 2016, con predominio de lesiones óseas en sexo masculino, edades jóvenes, ocupación comerciante (Mora, 2016). Se atribuye a que el sexo masculino en estas edades se expone más a trabajos en ocasiones en horarios nocturnos y con frecuencia de la necesidad de utilizar armas de fuego como medio de defensa o de ataque, además, de ser víctimas de asaltos, balas perdidas o violencia con utilización de éstas. Respecto a la región corporal, predominó en miembros inferiores con mayor frecuencia las fracturas de fémur en 20\% (19), según clasificación para fracturas expuestas de Gustilo y Anderson, se clasificó en G y A III-A el 21\% (17). Cabe resaltar que en los expedientes clínicos no especificaron ningún tipo de clasificación al momento del ingreso del paciente desde sala de emergencia 67\% (55), siendo de importancia para la decisión del tipo de tratamiento antibiótico según región anatómica y grado de contaminación.

Las fracturas por armas de fuego son lesiones traumáticas que afectan la productividad del paciente en la sociedad ya que se evidenció que los pacientes necesitaron tratamiento quirúrgico en el 90\% (74) y se presentó complicaciones el 37\% (30), siendo lesiones graves el 67\% (55), que por el tipo de fractura y complicaciones, el 54\% (44) fueron incapacitados para realizar sus trabajos diarios por más de un mes y permanentemente en el 2\% según Congreso de la República de Guatemala, no solo afectándose a ellos mismos sino a su núcleo familiar.

\section{Agradecimientos}

La investigación fue posible gracias al apoyo de las autoridades de la carrera Médico y Cirujano del Centro Universitario de Oriente en conjunto con el Hospital Regional de Zacapa.

\section{Referencias bibliográficas}

Baires, R. (2014). En Guatemala hay un arma por cada 25 habitantes. Plaza Pública. Recuperado de https:/www.plazapublica.com.gt/content/en-guatemala-hay-un-arma-por-cada25-habitantes

Comisión Internacional Contra la Impunidad en Guatemala -CICIG. (2009). Armas de fuego y municiones en Guatemala: mercado legal y tráfico ilícito. Recuperado de http://cicig.org/uploads/documents/informes/INFORTEMA_DOC01_20091201_ES.pdf 
Kuyigwa, T., Bitum, A. \& Longombe, O. (2015). Fractures by firearms in conflict town. Open Journal of Orthopedics 5:120-125. https://doi.org/10.4236/ojo.2015.55016

Mora, A. (2016). Incidencia de lesiones del aparato locomotor por heridas de arma de fuego. Tesis M.Sc. Guatemala, USAC, Facultad de Ciencias Médicas. Recuperado de http://biblioteca.usac.edu.gt/ tesis/05/05_10121.pdf

Palma, W. (2011). Consideraciones ortopédicas en las lesiones causadas por proyectil de arma de fuego en menores de edad. Revista Ortho-tips 7(3-4):135-141. Recuperado de http:/www.medigraphic.com/ pdfs/orthotips/ot-2011/ot113-4c.pdf

Pérez, E. (2003). Armas de fuego. Monografías. Recuperado de http://www.monografias.com/ trabajos13/ trarmas/trarmas. Shtml

Rodríguez, J. (2011). Fracturas por proyectil de arma de fuego en huesos largos de la extremidad pélvica. Revista Ortho-tips 7(3-4):147-154. Recuperado de http:/www.medigraphic.com/pdfs/orthotips/ot2011/ot113-4e.pdf

\section{Sobre el autor}

\section{Welner Guillermo Figueroa Chang}

Médico y Cirujano, egresado del Centro Universitario de Oriente, CUNORI, de la Universidad de San Carlos de Guatemala en el año 2018. Realizó el ejercicio de práctica hospitalaria en el Hospital Regional de Zacapa y EPS en clínica médica Iglesia Vida Real, Chiquimula en el año 2017. Ha realizado las siguientes investigaciones: "Uso adecuado de hilos de sutura en la emergencia de adultos del Hospital Regional de Zacapa” (2015), "Hallazgos electrocardiográficos en recién nacidos diagnosticados con asfixia perinatal” (2016) y "Caracterización de microorganismos aislados en cultivos de pacientes con infección de sitio quirúrgico" (2017), "Caracterización de pacientes con fracturas producidas por proyectil de arma de fuego" (2018).

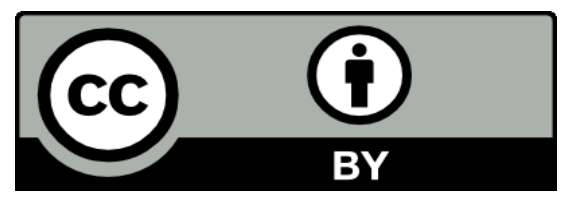

Este texto está protegido por una licencia CreativeCommons 4.0.

Usted es libre para compartir, copiar y redistribuir el material en cualquier medio o formato y adaptar el documento, remezclar, transformar y crear a partir del material para cualquier propósito, incluso comercialmente, siempre que cumpla la condición de atribución: usted debe reconocer el crédito de una obra de manera adecuada, proporcionar un enlace a la licencia, e indicar si se han realizado cambios. Puede hacerlo en cualquier forma razonable, pero no de forma tal que sugiera que tiene el apoyo del licenciante o lo recibe por el uso que hace. 